\title{
ANÁLISE DE FALHA DE UM COMPONENTE ESTRUTURAL DE PLANTA DE PELOTIZAÇÃO DE MINÉRIO DE FERRO
}

\author{
Marcelo Tadeu Milan ' \\ Omar Maluf ${ }^{2}$ \\ José Ricardo Tarpani ${ }^{3}$ \\ Claudio Finamore Ferraz ${ }^{4}$ \\ Jael Moutinho ${ }^{5}$ \\ Tenório Freitas ${ }^{6}$
}

\section{Resumo}

Barras de grelha de carros de pelotização de minério de ferro apresentaram intenso desgaste por abrasão devido ao contato com cargas de pelotas, e por erosão resultante do fluxo de ar quente contendo partículas do minério. A queima de combustível fóssil aliada à presença de contaminantes na carga de pelotas originaram eutéticos com baixo ponto de fusão de sais de metais alcalinos. A deposição de sais líquidos sobre a superfície das barras de grelha destruiram camada de óxidos protetores e induziram a degradação dos componentes por corrosão a quente. Descamação acelerada por fadiga térmica agravou sensivelmente o processo. Recomenda-se adotar um aço inoxidável com maiores teores de $\mathrm{Cr}$ para suprir a região empobrecida junto à superfície, e de Si e Al para aumentar a resistência global do aço à oxidação.

Palavras-chave: Aços inoxidáveis; Análise de falhas; Corrosão; Desgaste.

\section{FAILURE ANALYSIS OF STRUCTURAL COMPONENT OF IRON ORE PELLETIZING PROCESS PLANT}

\begin{abstract} global oxidation resistance.

Key words: Stainless steel; Failure analysis; Corrosion; Wear.

\section{INTRODUÇÃO}

Um esquema de parte de um forno de pelotização de minério de ferro é ilustrado na Figura la. Basicamente, uma correia sem fim, composta por vários carros, conduz pelotas através do forno. No fundo de cada carro há um arranjo de barras de grelha (indicado por uma seta azul) confeccionadas com aço inoxidável,
\end{abstract}

Grate bars of pallet cars employed in iron ore pelletizing process presented severe abrasive wear due to the contact with the pellets, and erosive wear as a consequence of the hot air flow containing iron ore particles. Burning fossil fuels and the presence of contaminants in the pellets resulted in low melting point eutectics of metal alkali salts. Liquid salt deposits on the grate bar surface dissolved the protective oxide layer, inducing hot corrosion degradation of the component. Thermal fatigue induced-spalling further aggravated the problem. It was recommended a higher $\mathrm{Cr}$-content stainless steel in order to supply the near surface $\mathrm{Cr}$-depleted zone as well as higher levels of $\mathrm{Si}$ and $\mathrm{Al}$ aimed at increasing

cuja função é sustentar a carga de pelotas sobre o carro e permitir a passagem de ar quente durante as etapas de queima do processo de pelotização. Um típico carro de grelha do tipo vagonete é ilustrado na Figura Ib, o qual circula ininterruptamente sobre trilhos.

'PhD em Engenharia, Diretor Superintendente MIB - Materials Institute of Brazil. Rua Paulino Botelho de Abreu Sampaio, 88I, Jd Bethânia, 1356I-060, São Carlos-SP. milan@mib.eng.br

${ }^{2}$ Doutor em Engenharia, Diretor Executivo MIB - Materials Institute of Brazil. Rua Paulino Botelho de Abreu Sampaio, 88I, Jd Bethânia, I356I-060, São Carlos-SP. omar@mib.eng.br

${ }_{3}^{3}$ Professor Associado do Departamento de Engenharia de Materiais, Aeronáutica e Automobilística, Escola de Engenharia de São Carlos, Universidade de São Paulo (SMM-EESC-USP). Av. Trabalhador São-Carlense, 400, Pq. Arnold Schimidt, 13566-590, São Carlos-SP. jrpan@sc.usp.br ${ }^{4}$ Engenheiro Mecânico Especialista em Materiais do Departamento de Engenharia de Materiais da CVRD, DIPE - Engenharia de Materiais, Prédio Oficina Elétrica, Térreo, Av. Dante Michelini, 5500, Ponta de Tubarão, 29090-900, Vitória-ES. claudio.ferraz@vale.com

${ }^{5}$ Técnico de Processo do Departamento de Gestão Estratégica de Insumos da CVRD, DIPE - Engenharia de Materiais, Prédio Oficina Elétrica, Térreo, Av. Dante Michelini, 5500, Ponta de Tubarão, 29090-900,Vitória-ES. jael.moutinho@vale.com

${ }^{6}$ Supervisor Técnico de Manutenção do Departamento de Pelotização da CVRD, DIPE - Engenharia de Materiais, Prédio Oficina Elétrica, Térreo, Av. Dante Michelini, 5500, Ponta de Tubarão, 29090-900,Vitória-ES. tenorio.freitas@vale.com 

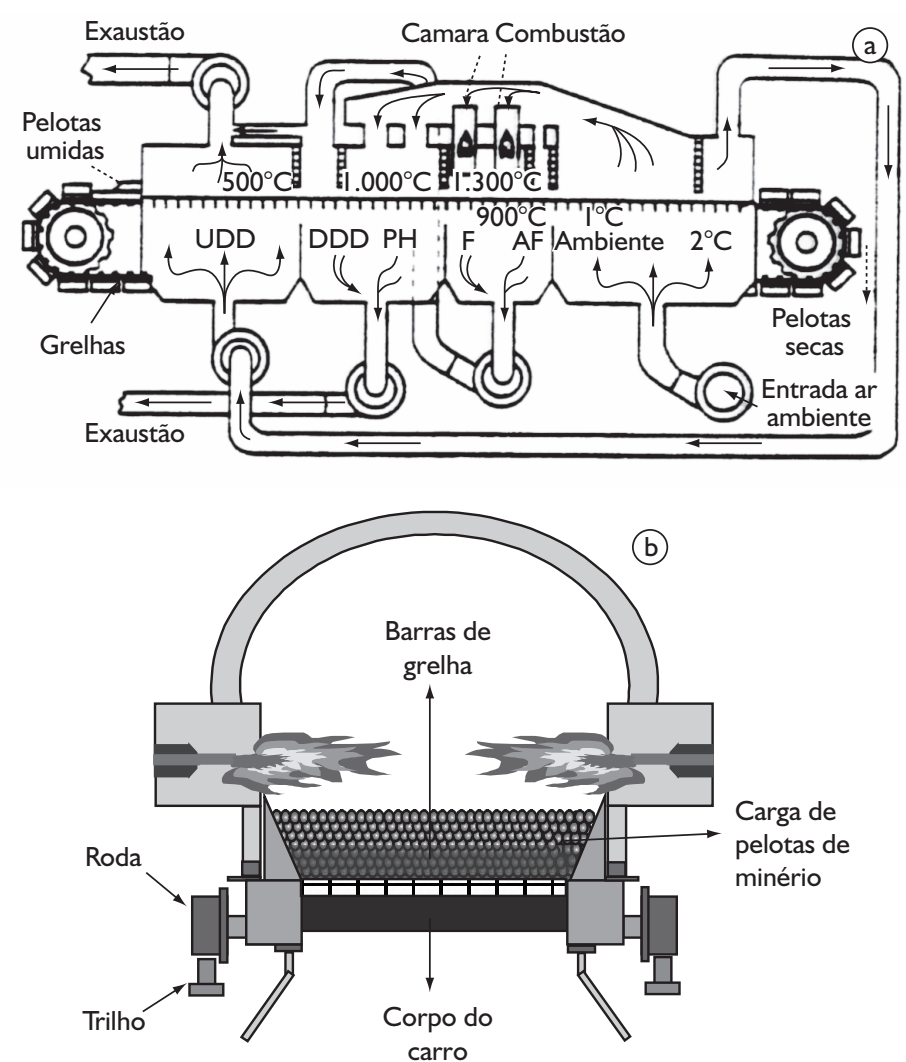

Figura I. a) Esquematização simples de forno de pelotização; e b) carro de grelha.

No presente trabalho, objetiva-se determinar os mecanismos de falha de barras de grelha utilizadas em carros de pelotização de minério de ferro, as quais apresentaram excessivo desgaste em serviço.

A Figura 2a mostra barras de grelha montadas sobre os carros, enquanto a Figura $2 \mathrm{~b}$ exibe dois destes componentes, um intacto e outro retirado de serviço.
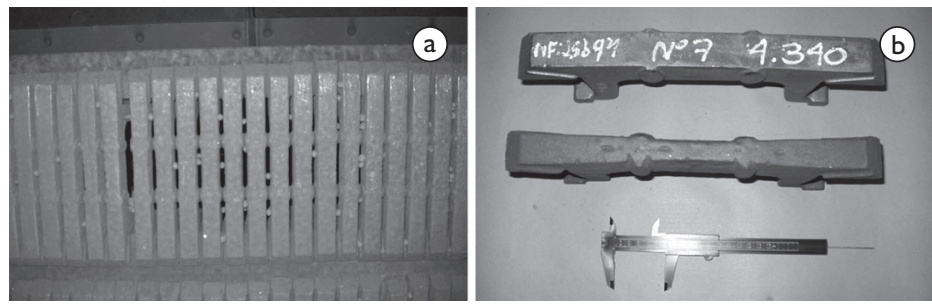

Figura 2. a) Barras de grelha montadas sobre um carro de pelotização; e b) amostras de barras intactas (acima) e já utilizadas em serviço (abaixo).

As barras de grelha estão sujeitas a ciclos de aquecimento seguidos de resfriamento que duram cerca de 40 minutos. A temperatura máxima atingida na queima, próximo às barras de grelha, é de cerca de $450^{\circ} \mathrm{C}$ em regime normal de operação. Entretanto, em paradas curtas para troca de carros, a temperatura do componente pode atingir até $650^{\circ} \mathrm{C}$.

A atmosfera do forno é oxidante e o aquecimento é propiciado por queima de óleo e/ou gás natural.

Os principais tipos de danos visualmente detectados no material são desgastes, vazios e empenamentos.

\section{EXPERIMENTAL}

Foram realizados os seguintes procedimentos:

- Análise química (espectrometria de emissão óptica e microsonda EDX);

- Análise visual;

- Análise por microscopias óptica e eletrônica de varredura (MEV);

- Macrodureza Brinell; e

- Análise química de sub-produtos de corrosão.

\section{RESULTADOS E DISCUSSÃO}

\section{I Análise Química do Componente}

Os resultados apresentados na Tabela I indicam que os materiais que constituem as barras de grelha, mostradas na Figura 2, encontram-se dentro dos valores especificados para o aço ASTM A297-HH, que é estabelecido em projeto.

Tabela I. Análise química por espectrometria de emissão óptica.

\begin{tabular}{lrrl}
\hline \multicolumn{1}{c}{ Barra } & Nova & Usada & A297-HH \\
\hline $\mathrm{C}$ & 0,22 & 0,40 & $0,20-0,50$ \\
$\mathrm{Si}$ & 1,19 & 0,92 & 2,0 máx \\
$\mathrm{Mn}$ & 1,20 & 1,07 & 2,0 máx \\
$\mathrm{P}$ & 0,03 & 0,03 & 0,04 máx \\
$\mathrm{S}$ & 0,02 & 0,03 & 0,04 máx \\
$\mathrm{Cr}$ & 25,20 & 24,00 & $24,0-28,0$ \\
$\mathrm{Ni}$ & 11,50 & 12,30 & $11,0-14,0$ \\
$\mathrm{Mo}$ & 0,20 & 0,15 & 0,50 máx \\
\hline
\end{tabular}

\subsection{Dureza}

Medidas de dureza Brinell com carga de $187,5 \mathrm{kgf}$ e esfera de $2,5 \mathrm{~mm}$ foram realizadas na seção transversal das barras mostradas na Figura 2, e os resultados são listados na Tabela 2.

Observa-se que os valores de dureza obtidos para a barra intacta e para a retirada de serviço são similares, e encontram-se bem abaixo do limite inferior da dureza de partículas de minério de ferro, que varia entre $400 \mathrm{HB}$ e $600 \mathrm{HB}$.

Tabela 2. Medidas de dureza Brinell efetuadas em plano transversal das barras recebidas para análise.

\begin{tabular}{ccccccc}
\hline Barra & \multicolumn{5}{c}{ Dureza HB } & Média \\
\cline { 2 - 6 } & $\mathbf{1}^{\circ}$ & $\mathbf{2}^{\circ}$ & $\mathbf{3}^{\circ}$ & $\mathbf{4}^{\circ}$ & $\mathbf{5}^{\circ}$ & \\
\hline Barra nova & 239 & 239 & 239 & 211 & 211 & 228 \\
Barra usada & 219 & 226 & 219 & 229 & 223 & 223 \\
\hline
\end{tabular}




\subsection{Análise Visual}

A macro-análise da barra já usada em serviço apresenta uma camada de óxidos generalizada sobre a superfície (Figura 3). Macroscopicamente, não foram observadas trincas superficiais, somente alguns defeitos de fundição (vazios) e regiões com perda de massa mais acentuada. Foram estimadas perdas de massa da ordem de ate $26 \%$ relativamente às barras virgens.
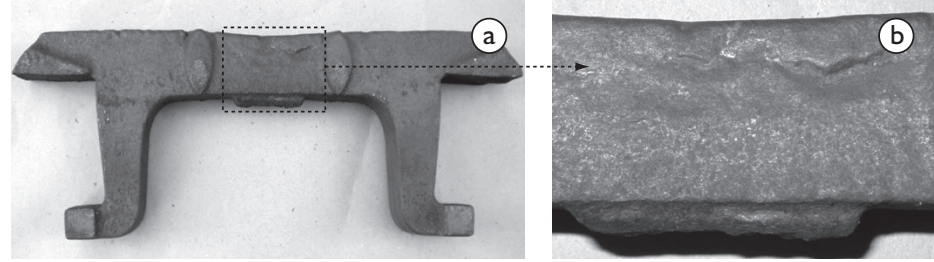

Figura 3. a) Aspecto da barra de grelha usada mostrando a camada de óxidos formada sobre a superfície durante a operação em serviço; e b) detalhe de um vazio gerado na fundição.

Da Figura 2, já ficara claro que a exposição ao ambiente de serviço provoca perda de massa, reduzindo de maneira significativa a largura da barra de grelha, especialmente no centro, em função do contato com a carga de pelotas e maior fluxo local de ar.

\subsection{Análises por Microscopia Óptica e EDX}

A análise metalográfica do centro da barra de grelha virgem apresenta uma microestrutura típica de aço inoxidável $\mathrm{HH}$, contendo, inclusive, carbetos de cromo precipitados em contornos de grão austeníticos (regiões interdendríticas na Figura 4a). Por outro lado, a metalografia de amostra do centro da barra de grelha já utilizada em serviço (Figura 4b) mostra extensivo coalescimento de carbetos primários e intensa precipitação de carbetos secundários. $\mathrm{A}$ austenita pode reter até $0,2 \%$ de carbono em solução sólida, porém, em situações exposição do material por longo tempo a altas temperaturas, o carbono pode se combinar com o cromo, ou outros elementos de liga para formar uma fina distribuição de carbetos na microestrutura.
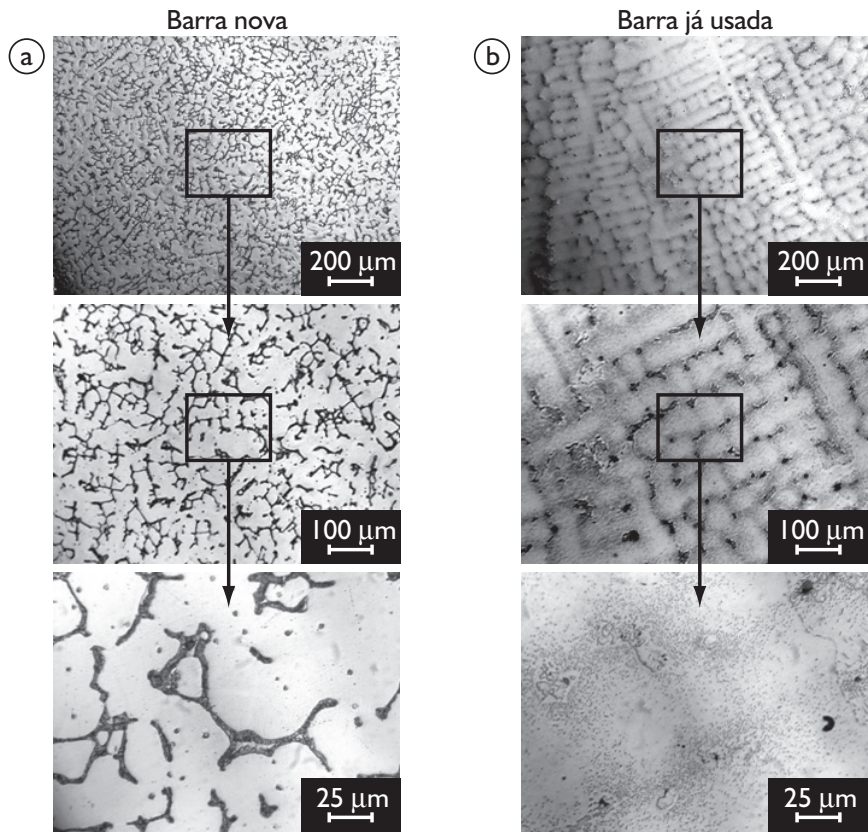

Figura 4. Análise micrográfica do plano transversal das barras mostrando a presença de carbetos em regiões interdendríticas (regiões mais escuras).
$\mathrm{Na}$ Figura 5, a análise metalográfica de região próxima à superfície da barra de grelha usada mostra a presença de três regióes distintas.

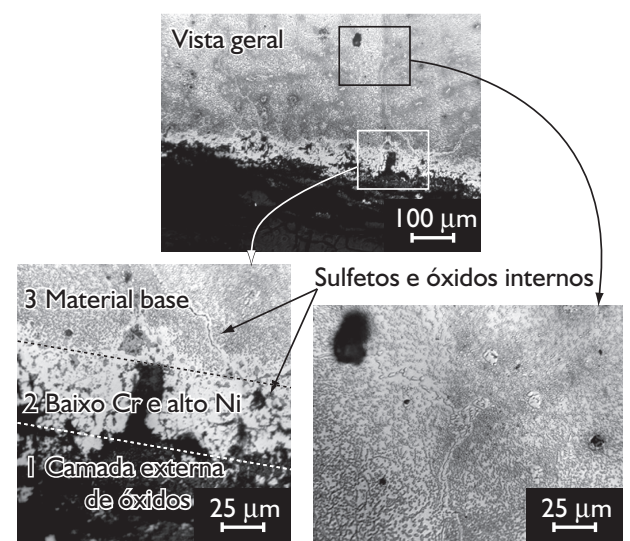

Figura 5. Análise metalográfica da superfície da barra de grelha usada mostrando as três principais regiões: I) camada externa de óxidos, 2) região de depleção de $\mathrm{Cr}$, e 3) metal base.

A primeira consiste de uma camada de óxidos fragmentada, porosa e provavelmente não-protetora. A análise por EDX dos pontos identificados na Figura 6 sugere a presença predominante de $\mathrm{Cr}_{2} \mathrm{O}_{3}, \mathrm{Fe}_{2} \mathrm{O}_{3}$ e/ou espinéis de $\mathrm{Fe}-\mathrm{Cr}$, conforme evidenciam os dados apresentados na Tabela 3.

A segunda região consiste do metal adjacente à camada de óxidos, que apresenta algumas trincas, vazios e óxidos/sulfetos internos, com pouca ou nenhuma precipitação de carbetos. A análise por microsonda EDX revelou que esta região é empobrecida em $\mathrm{Cr}$ e rica em $\mathrm{Ni}$. Foram encontradas grandes quantidades de sulfetos de cromo, provavelmente do tipo $\mathrm{Cr}_{3} \mathrm{~S}_{4}$ ou $\mathrm{CrS}$. A depleção do teor de cromo da liga, próximo à superfície, pela formação de óxidos e sulfetos, torna o material suscetível ao ataque corrosivo, uma vez que o caráter inoxidável do aço requer um teor mínimo de $12 \% \mathrm{Cr}$. O enxofre é proveniente da queima dos combustíveis utilizados no processo e da contaminação da carga de pelotas.

Por fim, na terceira região, a partir de cerca de $100 \mu \mathrm{m}$ da superfície, observa-se uma intensa precipitação de carbetos, sem sinais de oxidação interna, com a composição química mantendo-se próxima à da liga base, conforme demonstra a análise por EDX na Tabela 3.

Tabela 3. Análise semiquantitativa por EDX dos pontos I-4 da Figura 6 (\% em massa aproximada).

\begin{tabular}{lcccc}
\hline & Ponto I & Ponto 2 & Ponto 3 & Ponto 4 \\
\hline $\mathrm{Fe}$ & 39 & 50 & 10 & 64 \\
$\mathrm{Cr}$ & 38 & $\mathrm{I}$ & 60 & 23 \\
$\mathrm{Ni}$ & 6 & 49 & 4 & 12 \\
$\mathrm{Mn}$ & 0,8 & $0, \mathrm{I}$ & 0,6 & 0,5 \\
$\mathrm{Si}$ & 1,6 & $0, \mathrm{I}$ & 2,2 & 0,7 \\
$\mathrm{~S}$ & - & $0, \mathrm{I}$ & 8,9 & - \\
$\mathrm{O}$ & 15 & - & 15 & - \\
$\mathrm{Cl}$ & - & - & - & - \\
\hline
\end{tabular}




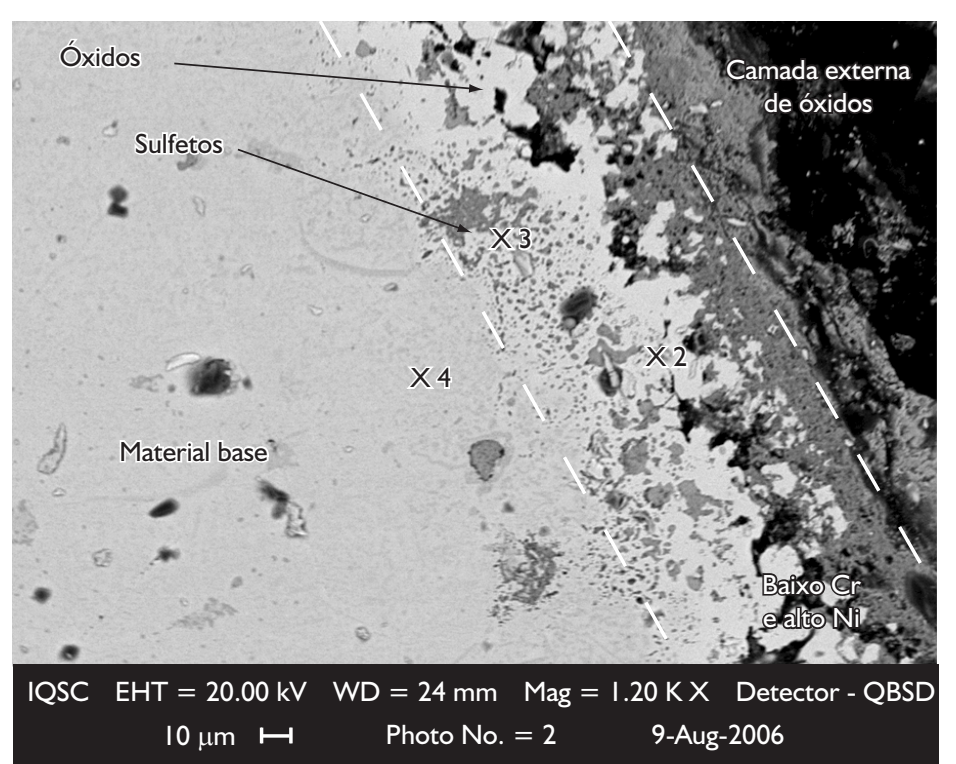

Figura 6. Pontos analisados por EDX junto à superfície da barra de grelha utilizada em serviço.

Apesar da análise por EDX da amostra metalográfica não ter apontado a presença do elemento químico $\mathrm{Cl}$, este foi identificado em alguns pontos da superfície da barra de grelha usada, conforme indicado na Tabela 4. Embora, $\circ \mathrm{Cl}$ possa ter sido proveniente do próprio manuseio da barra de grelha, não se pode descartar a possibilidade de que este elemento esteja presente na carga de pelotas na forma de sais de metais alcalinos $(\mathrm{Na}, \mathrm{K})$ e alcalino-terrosos $(\mathrm{Mg}, \mathrm{Ca})$, provenientes dos aditivos que são adicionados para a queima ou até mesmo advindos da atmosfera marinha na qual o forno se encontra. Além disto, a presença detectada de enxofre pode indicar a existência de sais do tipo sulfato.

Tabela 4. Análise semiquantitativa por EDX da superfície da barra de grelha utilizada em serviço. (\% em peso aproximado).

\begin{tabular}{ccccccccc}
\hline & $\mathbf{F e}$ & $\mathbf{C r}$ & $\mathbf{N i}$ & $\mathbf{M n}$ & $\mathbf{S i}$ & $\mathbf{S}$ & $\mathbf{O}$ & $\mathbf{C l}$ \\
\hline Ponto I & 36 & 18 & 4 & 10 & $\mathrm{I}, 6$ & 0,9 & 27 & $0, \mathrm{I} *$ \\
Ponto 2 & 37 & 17 & 10 & 2 & 0,8 & 0,4 & $\mathrm{I}, 4 *$ & 10 \\
\hline
\end{tabular}

* Valores dentro do erro experimental das medições por EDX.

Visando confirmar a presença de sais na superfície da barra de grelha usada, procedeu-se à análise química, por colorimetria, dos sub-produtos aderidos à superfície. A amostra foi lavada com uma solução de água destilada com $1 \%$ de $\mathrm{HNO}_{3}$ para a solubilização dos sais presentes. Pelos resultados apresentados na Tabela 5 , pode-se verificar uma pequena presença de ânions cloreto $\left(\mathrm{Cl}^{-}\right)$e sulfato $\left(\mathrm{SO}_{4}{ }^{2-}\right)$, além dos cátions de metais alcalinos $\mathrm{Na}^{+}$e K ${ }^{+} \mathrm{e}$ metais alcalino-terrosos $\mathrm{Mg}^{2+}$ e $\mathrm{Ca}^{2+}$. A predominância é de sais do tipo sulfato de sódio. $O$ teor total de sais encontrado sobre a superfície foi de aproximadamente $0,3 \mathrm{mg} / \mathrm{cm}^{2}$.

Tabela 5. Análise química dos sub-produtos de corrosão depositados sobre a superfície da barra de grelha usada. Valores aproximados.

\begin{tabular}{lccccccc}
\hline & \multicolumn{4}{c}{ Cátions } & & \multicolumn{2}{c}{ Ânions } \\
\cline { 2 - 3 } & $\mathbf{N a}^{+}$ & $\mathbf{K}^{+}$ & $\mathbf{C a}^{+2}$ & $\mathbf{M g}^{+2}$ & & $\mathbf{S O}^{-2}$ & $\mathbf{C l}^{-}$ \\
\hline Concentração (mg/L) & 15 & 2 & 0,4 & 0,0 & & 10 & $\mathrm{I}$ \\
Fração Molar (\%) & 91 & 8 & $\mathrm{I}, 5$ & 0,2 & & 88 & 12 \\
\hline
\end{tabular}

As características da morfologia da corrosão observada, i.e. camada de óxidos porosa e fragmentada junto com sulfetos internos, são consistentes com processos de corrosão a quente induzidos por sais dos tipos sulfatos e cloretos de metais alcalinos e alcalino-terrosos. ${ }^{(1-3)}$ A mistura destes sais e óxidos pode resultar na geração de eutéticos de baixo ponto de fusão, da ordem de $500^{\circ} \mathrm{C}$, os quais podem depositar-se no estado líquido sobre a superfície das barras em serviço, propiciando corrosão a quente, uma vez que a temperatura das barras pode exceder os $600^{\circ} \mathrm{C}$ durante eventuais paradas curtas do forno. A presença de sais líquidos destrói a camada de óxidos protetores por meio de sua dissolução.

Não obstante os resultados da análise por microscopia óptica indiquem que o principal mecanismo de degradação da liga é por oxidação/ corrosão a quente, a perda de massa do material ocorre por meio de 3 mecanismos distintos, os quais são responsáveis pela remoção da camada de óxidos, expondo a superfície intacta do metal a novos ataques. São eles:

- Desgaste por abrasão propiciado pelo contato e movimento relativo da carga de pelotas sobre as barras de grelha durante as etapas de carregamento e descarregamento;

- Desgaste erosivo provocado pelo impacto de partículas de minério em suspensão no fluxo de ar ascendente e/ ou descendente;

- Fadiga térmica provocada pelos ciclos de resfriamento e aquecimento das barras de grelha. As diferenças dos coeficientes de expansão térmica e de volumes molares entre a camada de óxidos e - metal base introduzem tensões na camada de óxidos, fragmentando-a (spalling).

\section{PRINCIPAIS CONCLUSÕES}

Os resultados obtidos no presente estudo permitem concluir que:

- O material analisado encontra-se dentro das especificações de composição química estabelecidas para o aço ASTM A297-HH;

- O dano é introduzido no material por mecanismos de oxidação em altas temperaturas e corrosão a quente devido à presença de sais na carga de pelotas e/ou provenientes da atmosfera marinha; 
- A perda de massa ocorre por meio de uma sinergia entre os processos de desgaste abrasivo, desgaste erosivo e fadiga térmica (spalling).

\section{RECOMENDAÇÕES}

De modo a evitarem-se os problemas ora reportados em futuros componentes em serviço, são recomendadas as seguintes ações preventivas: a) Utilizar liga com teor de cromo mais elevado, de modo a aumentar o suprimento deste elemento na região depletada junto à superfície;

b) Especificar uma liga com teores de $\mathrm{Si}$ e Al mais elevados visando aumentar a sua resistência global à oxidação.

\section{REFERÊNCIAS}

I KHANNA, A.S. Oxidation of alloys. In: Materials Park, OH, USA: ASM Intl 2002. p. I I0-34.

2 KHANNA, A.S. Hot corrosion. In: rials Park, OH, USA: ASM Intl, 2002. p. I72-20I.

3 MOHANTY, B.P.; SHORES, D.A. Hot corrosion of stainless steel grate bars in taconite indurators. In: Handbook of case histories in failure analysis. Carol Stream, II, USA: ASM IntI, I992. v. I, p.358-62.

Recebido em: 24/03/08

Aceito em: 15/08/08

Proveniente de: CONGRESSO ANUAL DA ABM, 62., 2007, Vitória, ES. São Paulo: ABM, 2007. 